\title{
The Effect of Truncation on Very Small Cardiac SPECT Camera Systems
}

\author{
Damien Rohmer ${ }^{1}$, Robert L. Eisner ${ }^{2}$, and Grant T. Gullberg ${ }^{1}$ \\ ${ }^{1}$ Lawrence Berkeley National Laboratory, 1 Cylotron Road Berkeley, CA 94720 \\ ${ }^{2}$ Emory Crawford Long-Hospital, 550 Peachtree St, NE Atlanta, GA 30308
}

August 1, 2006

\begin{abstract}
Background: The limited transaxial field-of-view (FOV) of a very small cardiac SPECT camera system causes view-dependent truncation of the projection of structures exterior to, but near the heart. Basic tomographic principles suggest that the reconstruction of non-attenuated truncated data gives a distortion-free image in the interior of the truncated region, but the DC term of the Fourier spectrum of the reconstructed image is incorrect, meaning that the intensity scale of the reconstruction is inaccurate. The purpose of this study was to characterize the reconstructed image artifacts from truncated data, and to quantify their effects on the measurement of tracer uptake in the myocardial. Particular attention was given to instances where the heart wall is close to hot structures (structures of high activity uptake).

Methods: The MCAT phantom was used to simulate a 2D slice of the heart region. Truncated and non-truncated projections were formed both with and without attenuation. The reconstructions were analyzed for artifacts in the myocardium caused by truncation, and for the effect that attenuation has relative to increasing those artifacts.

Results: The inaccuracy due to truncation is primarily caused by an incorrect DC component. For visualizing the left ventricular wall, this error is not worse than the effect of attenuation. The addition of a small hot bowel-like structure near the left ventricle causes few changes in counts on the wall. Larger artifacts due to the truncation are located at the boundary of the truncation and can be eliminated by sinogram interpolation. Finally, algebraic reconstruction methods are shown to give better reconstruction results than an analytical filtered back-projection reconstruction algorithm.

Conclusion: Small inaccuracies in reconstructed images from small FOV camera systems should have little effect on clinical interpretation. However, changes in the degree of inaccuracy in counts from slice to slice are due to changes in the truncated structures. These can result in a visual 3-dimensional distortion. As with conventional large FOV systems attenuation effects have a much more significant effect on image accuracy.
\end{abstract}

keywords: Truncation, Cardiac Imaging, SPECT.

\section{Introduction}

$\mathrm{W}$ HEN performing cardiac imaging using systems such as SPECT camera, truncation can occur due to the limited field-of-view. Artifacts due to the inconsistency of the data can then spread onto the heart-wall close to the truncated section. Tomographic principles show that the exact reconstruction of truncated projections cannot be performed $[2,3]$. However, the reconstruction gives an approximate intensity in the non truncated section and preserves high frequency structures.

The objective of this paper is to describe the arti- facts caused by the truncation and to quantify them on some sections of the heart-wall. The study endeavors to differentiate artifacts due to attenuation from those created by the truncation. Also, a study of the impact of a structure of high activity uptake (hot spot) situated close to the heart wall is performed. Analytical reconstructions can develop a strong artifact at the truncation boundary due to the discontinuity of the projected data. It is shown that this artifact can be eliminated by sinogram interpolation. Finally, a comparison between analytical and some iterative reconstruction methods are performed. 


\section{Material and Methods}

\section{$2.1 \quad$ Model}

A transaxial slice of an intensity map is taken from the Mathematical Cardiac-Torso (MCAT) phantom where the heart is close to the liver (see Fig. 1). The dimension of the map is $256 \times 256$ pixels with $0.23 \mathrm{~cm} / \mathrm{pixel}$ and the body torso is $18 \times 12 \mathrm{~cm}$.

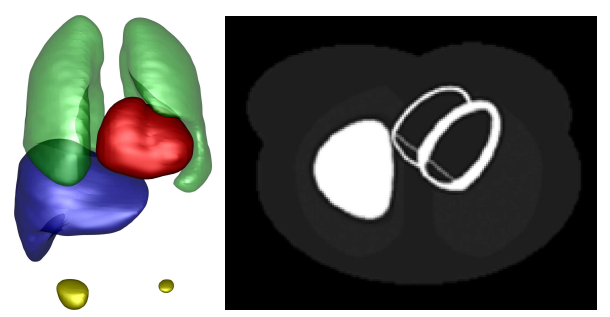

Figure 1: The picture on the left shows the distribution of the organs in the torso. A transaxial slice is taken where the heart is close to the liver. Its activity map is shown in the picture on the right.

To separate the effects of truncation and the effects of attenuation, an attenuation map was taken into account to simulate attenuated projections. This map was generated with the MCAT phantom assuming an attenuation of $140 \mathrm{KeV}$ for the transaxial slice shown in Fig. 2. The characteristics of the organs seen on the slice are given in Table 1 .
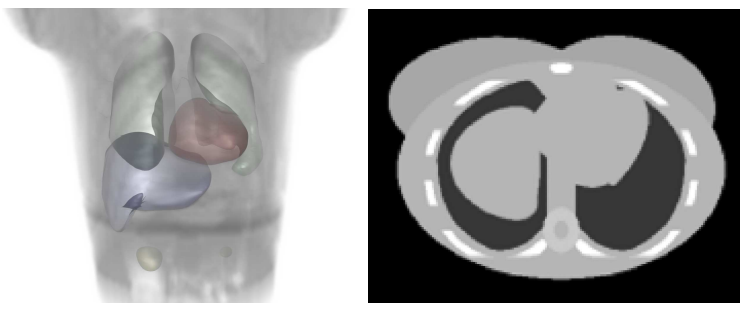

Figure 2: Attenuation map. The picture at the left shows the global attenuation distribution in the volume of the organs. The right picture is the slice of the selected attenuation map.

\subsection{Simulation of Projections}

The projections are simulated as the attenuated Radon transform of the selected slices of the MCAT, assuming parallel rays. Let $f$ be the activity map and $\mu$ the associated attenuation map. Then the projection $p$ is defined by:

$$
p_{\theta}(s)=\int_{t \in \mathbb{R}} f\left(s \boldsymbol{\theta}+t \boldsymbol{\theta}^{\perp}\right) e^{-\int_{t_{2}=t}^{+\infty} \mu\left(s \boldsymbol{\theta}+t_{2} \boldsymbol{\theta}^{\perp}\right) \mathrm{d} t_{2}} \mathrm{~d} t,
$$

Table 1: Characteristics of the activity and attenuation values for the organs in the slices

\begin{tabular}{|l|l|l|}
\hline Organ & $\begin{array}{l}\text { Activity (in } \\
\text { normalized } \\
\text { units) }\end{array}$ & $\begin{array}{l}\text { Attenuation } \\
\text { (/cm) }\end{array}$ \\
\hline Myocardium & 75 & 0.15 \\
Liver & 75 & 0.15 \\
Blood Pool & 2 & 0.15 \\
Fat & 2 & 0.238 \\
Lung & 4 & 0.04 \\
Spine & 2 & 0.17 \\
Rib Bone & 2 & 0.21 \\
Body & 2 & 0.15 \\
\hline
\end{tabular}

where $\boldsymbol{\theta}=\left(\begin{array}{r}-\sin (\theta) \\ \cos (\theta)\end{array}\right)$ and $\boldsymbol{\theta}^{\perp}=\left(\begin{array}{c}\cos (\theta) \\ \sin (\theta)\end{array}\right)$.

To model projection measurements at different camera positions, the projections were computed for an angle spanning $180^{\circ}$. The starting angle was positioned at $45^{\circ}$ right anterior oblique (RAO) as seen in Fig. 3. This information of angular sampling is less important for the unattenuated case where $p_{\theta+\pi}=p_{\theta}$, whereas, the artifacts due to attenuation depend on the sampling range.

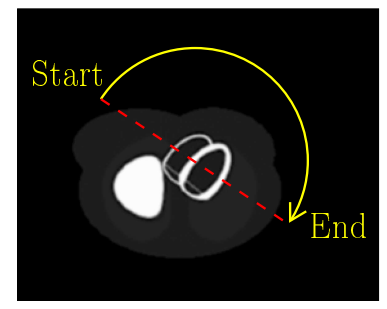

Figure 3: Rotation of the camera around the heart. The camera starts at $45^{\circ} \mathrm{ROA}$ and spans $180^{\circ}$.

A high angular sampling was performed by taking 400 projections during the rotation. This fine sampling avoids artifacts caused by insufficient angular sampling.

\subsection{Truncation}

The small field of view (FOV) of the camera truncates the projections from the complete activity map. Therefore some parts of the torso are only inside the field of view for some projections. The heart was situated in the middle of the FOV of the camera, so it could be visible from every angle. The region of interest (ROI) was taken small enough to just cover the heart geometry. The considered slice was then 
truncated around a circle of radius $r_{0}=7.6 \mathrm{~cm}$. This covered roughly $50 \%$ of the maximum body width as seen in Fig. 4.

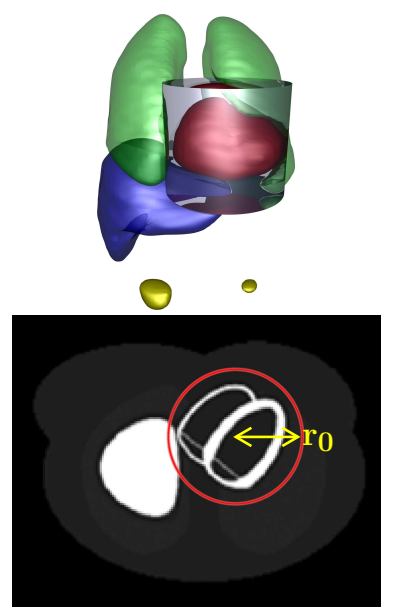

Figure 4: View of the Region Of Interest (ROI) covering the heart geometry. The picture of the left shows the ROI with the blue transparent cylinder for the geometry of the heart. Picture on the right shows the circle of truncation on the slice.

It was assumed that the camera had a perfect circulatory trajectory. The truncation was then modeled by keeping only the projection information of the sinogram for $|s| \in\left[0, r_{0}\right]$ (see Fig. 5). Then the truncated projection was denoted as $p_{\theta}^{T}$ such that:

$$
\begin{gathered}
p_{\theta}^{T}(s)=\left\{\begin{array}{ccc}
p_{\theta}(s) & \text { if } & |s| \leq r_{0} \\
0 & \text { if } & |s|>r_{0}
\end{array}\right. \\
\Rightarrow p^{T}(s)=p_{\theta}(s) \Pi\left(\frac{s}{2 r_{0}}\right) .
\end{gathered}
$$
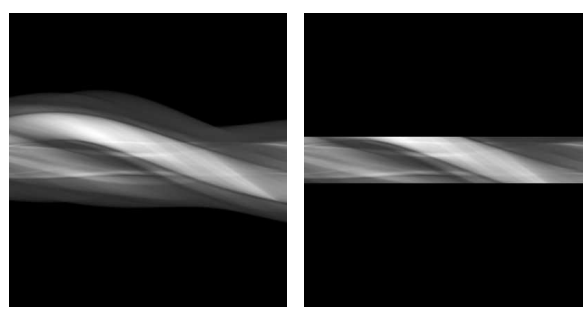

Figure 5: The picture on the left shows the complete sinogram before truncation, picture on the right shows the effect of the truncation on the projection data assuming the trajectory is perfectly circular.

\subsection{Reconstruction}

For the reconstruction, the Filtered Backprojection (FBP) without attenuation correction was used. The filtering was performed using the classical ramp filter without low pass filtering in order to obtain all of the artifacts including the high frequency artifacts.

Let us denote $r$ as the result of the reconstruction of the sinogram such that

$$
\left.r\left(p_{\theta}\right)=\mathcal{B}\left\{\mathcal{F}_{1 D}^{-1}\left\{|w| \mathcal{F}_{1 D}\left\{p_{\theta}\right\}\right\}\right\}\right\}
$$

where $\mathcal{B}\{f\}=\int_{\theta=0}^{\theta=\pi} f(x \cos (\theta)+y \sin (\theta)) \mathrm{d} \theta$; and $\mathcal{F}_{1 D}$ and $\mathcal{F}_{1 D}^{-1}$ are the one dimensional Fourier and inverse Fourier transform, respectively.

The reconstruction of the truncated sinogram is then equivalent to:

$r\left(p_{\theta}^{T}\right)=2 r_{0} \mathcal{B}\left\{\mathcal{F}_{1 D}^{-1}\left\{|w|\left[\mathcal{F}_{1 D}\left\{p_{\theta}\right\} * \operatorname{sinc}\left(2 r_{0} w\right)\right]\right\}\right\}$

\subsection{Hot Structure}

Finally, a study was performed by taking in account a hot bowel-like structure close to the left ventricle. This structure is shown in a gated acquisition of a real patient study in Fig. 6 A copy of this structure

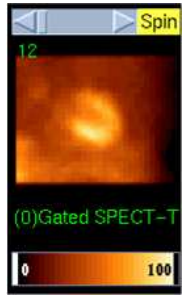

Figure 6: Acquisition of a patient study showing a hot structure (high activity) close to the left ventricle

is simulated with a maximal activity normalized to 70 (the myocardium is normalized to 75 as shown in Table 1). Moreover an attenuation factor of $0.175 / \mathrm{cm}$ is assumed for this shape. The two modified maps can be seen in Fig. 7. This structure is placed close to the left ventricle and positioned exactly at the limit of the truncated section, such that half of the structure is truncated.

\subsection{Analysis of the Data}

For each experiment, a qualitative visualization of the artifacts was performed by plotting the reconstructed activity map. A quantitative study was also done by sampling along some profiles. The study mainly focused on the left ventricle wall. For this purpose, a mask was created on the heart wall as seen in Fig. 8 

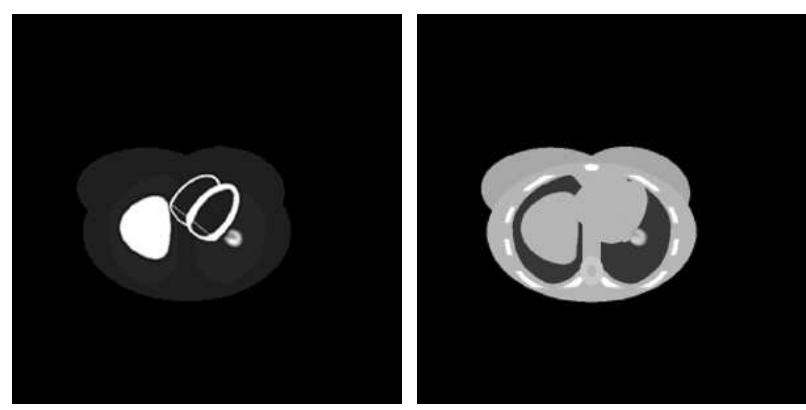

Figure 7: The addition of the hot structure. The left figure shows the activity map with the hot spot. The picture on the right is the attenuation map with the added structure.

and three profiles crossing this mask were defined. An oblique line (in yellow) crossed the septum and the other two lines crossed the anterior (blue) and posterior (green) lateral wall. Each line converged and passed through the added hot spot.
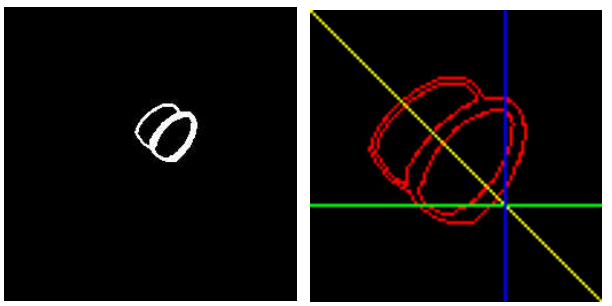

Figure 8: Figure on the left shows the mask used for the sampling in the heart wall. Figure on the right shows the sampling lines crossing the walls of the RV and LV.

\section{$3 \quad$ Results}

\subsection{No Attenuation}

\subsubsection{Artifact Visualization}

The first simulation took in account the normal activity map but without attenuation. A comparison was then performed between Filtered Backprojection (FBP) reconstruction of truncated and non truncated data.

The result is shown in Fig. 9. For the case of truncation, the intensity is scaled by a factor of 0.75 . A light ring circle is visible all around the boundary of the truncation. Except for close to the liver, the region outside of the ROI has almost a null value. Also, the basic shape and the homogeneity of the activity on the left ventricle seems to be unaffected by the truncation.
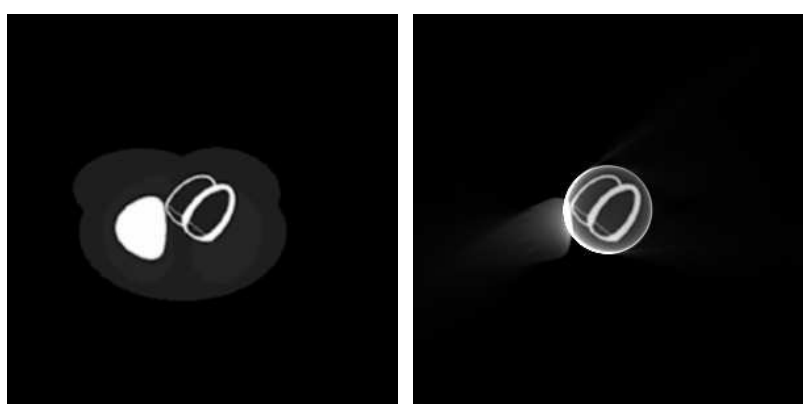

Figure 9: Results of the back projection of the activity map for the non attenuated case. Picture on the left is the non truncated reconstruction and the picture on the right is the results of truncation. The intensity of the truncated picture is scaled by a factor of 0.75 .

\subsubsection{Artifact Quantification}

The artifacts were then quantified by taking some samples of the reconstructed counts along the lines shown in Fig. 8 An example of samples is shown for the first line in Fig. 10. The sampling is done for the non truncated reconstruction (black) and the truncated reconstruction (dashed blue). The counts are especially comparable inside the heart wall (boundaries shown by the dashed red lines). The counts were normalized to one for the maximum activity (liver) in the non truncated case. The two other profiles of the samples are shown on Fig. 11. For all the samples, the truncation causes a global increase of the counts inside the ROI with a boundary marked by a high intensity in the picture. However, the change in the reconstructed activity is not significant.

\subsubsection{Component}

The majority of the problem seems actually to come from a change in the average value of counts inside of the ROI. The total amount of counts is increased by an unknown factor. This increase of counts is primarily due to the filtered backprojection reconstruction algorithm. In the reconstruction process, the method assumes that all of the counts are coming from the interior of the ROI. Thus, the increase of counts coming, for instance from the liver, are constrained to be backprojected inside this ROI. Therefore, the interior counts are higher than what it should be.

A global coefficient $\mu=0.85$ applied to the truncated reconstruction brings the counts closer to what is the true count level. Fig. 12 shows the profiles for the sampling along the three lines where the entire truncated reconstruction was scaled by $\mu$.

For the entire heart wall, the average error due to truncation was calculated. Denoting \#heart as the 


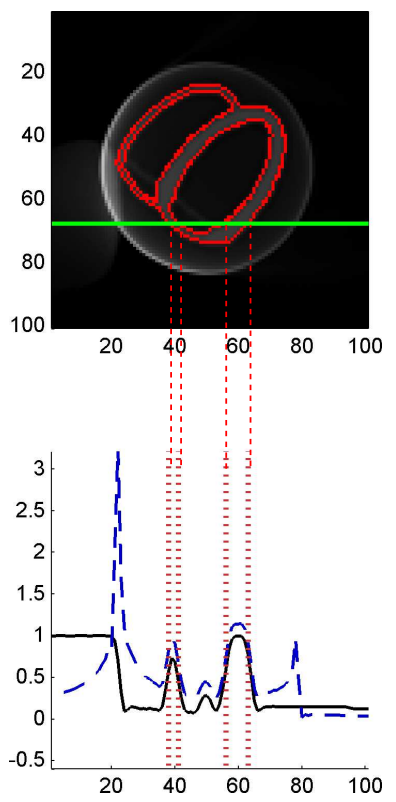

Figure 10: Example of sampling for the corresponding position through the heart. The black line is the sampling on the filtered backprojection reconstruction of the non truncated activity map. The dashed blue line corresponds to the truncated reconstruction and the dashed red line delineates the position of the heart wall.

number of pixels inside the heart wall, the averaged count error was calculated using:

$$
d_{1}=\frac{1}{\text { \#heart }} \sum_{\text {pixel } \in \text { heart }}\left|r\left(p_{\theta}^{T}\right)-r\left(p_{\theta}\right)\right|
$$

This result was compared to the case with the global correction coefficient:

$$
d_{2}=\frac{1}{\# \text { heart }} \sum_{\text {pixel } \in \text { heart }}\left|\mu r\left(p_{\theta}^{T}\right)-r\left(p_{\theta}\right)\right|
$$

In the first case, the averaged count difference is $d_{1} \simeq 0.17$ and in the second case $d_{2} \simeq 0.06$. Therefore the difference between the absolute counts can be decrease by more than a half.

The difference map is shown in Fig. 13 where the difference between the counts is shown for each pixel inside the heart wall. It appears that with the classical reconstruction, the entire surface of the heart wall has an increase in counts compared to the non truncated case. The maximum difference is close to the liver which introduces artifacts on the right ventricle. The same measurement but limited to the left ventricle gives $d_{1} \simeq 0.14$ and $d_{2} \simeq 0.04$, respectively.

The effect of truncation doesn't change the count ratio inside the same slice as the non linear change of

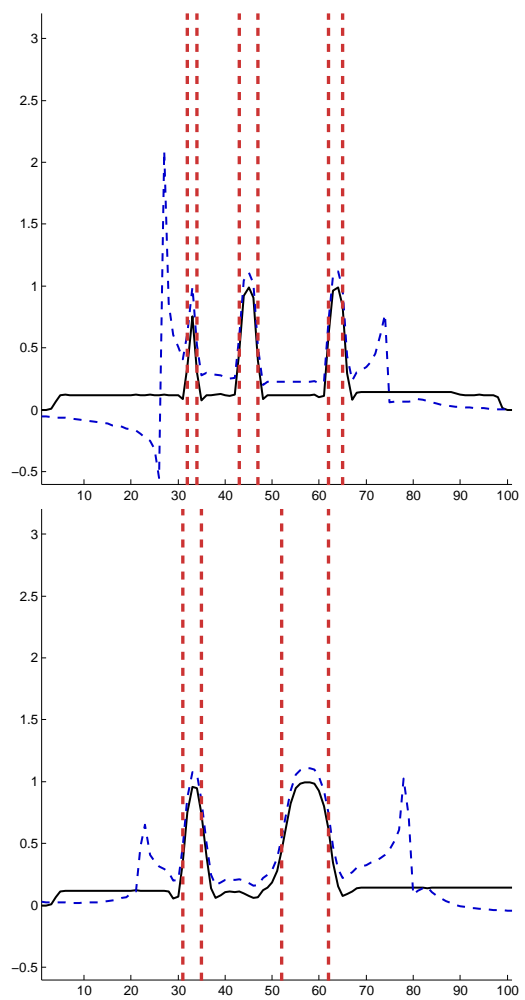

Figure 11: Samples along the diagonal line (top) and along the vertical line (bottom) is the dashed blue plot. The plot showing the black line is the reconstruction of the non truncated data.

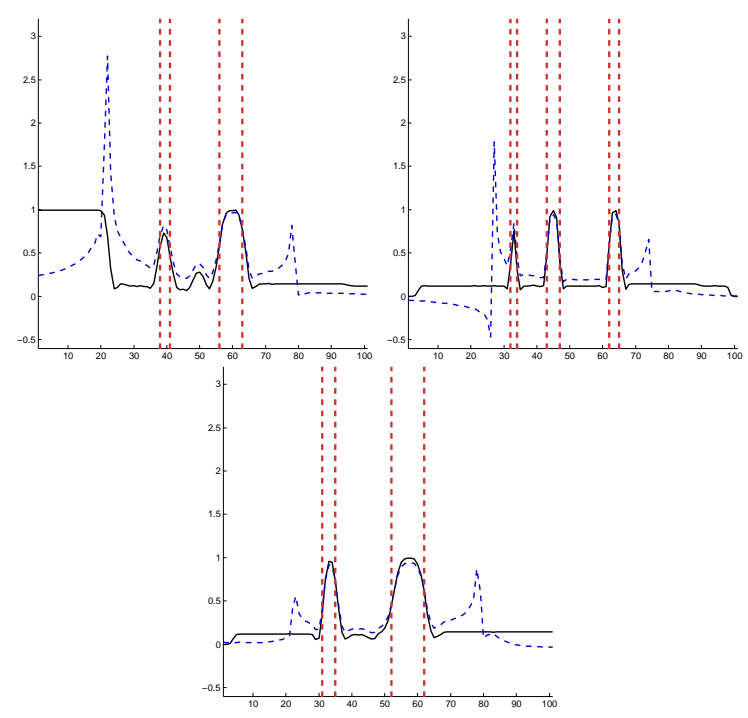

Figure 12: Samples along the horizontal and diagonal line (top-left), diagonal line (top-right) and along the vertical line (bottom) are shown as a dashed blue plot. The black line in the plots is the reconstruction of the non truncated data.

the absolute counts is small (less than the half of the 

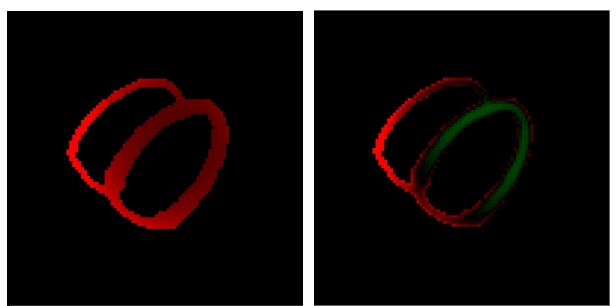

Figure 13: Comparison of the difference map for the non attenuated case. The figure at the left shows the error for the case of no scaling. The figure at the right shows the error for the case where the truncated reconstruction is multiplied with a homogeneous scaling factor. The red color indicates an increase of counts compared to the non truncated case and the green color a decrease. Each map has a color scaled to its maximum.

difference).

\subsection{With Attenuation}

A classical truncated reconstruction does not lead to a large error on the heart wall even if the ROI is close to the wall. However the previous study was performed without taking into account the effects of attenuation. Attenuation can spread artifacts in the direction of the rays. The goal of this study was to check if the attenuation factor makes the truncation artifacts worse. First of all, the comparison cannot be performed between the reconstruction of attenuated and non attenuated projections since the difference between the two is very large as seen in Fig. 14. The attenuation factor has a significant effect on the reconstructed picture due to large non-linear changes in the intensity distribution.

\subsubsection{Artifact Visualization}

The truncation artifacts for the attenuation case were then compared to the non truncated attenuated reconstruction. Fig. 15 shows the artifacts due to truncation. It can be seen that the ring artifact is still visible. Moreover, more artifacts are spread out from the ROI. The heart wall doesn't seem to have large changes compared to the non truncated case.

\subsubsection{Artifact Quantification}

The same technique used for quantification for the non attenuation case is used for the attenuation case (see sampling lines in Fig. 8). The three profiles are shown in Fig.16 The truncation produces globally the same effect for the attenuation case. The counts are increased inside the ROI without large changes in the activity in the heart. The ring artifact results in a
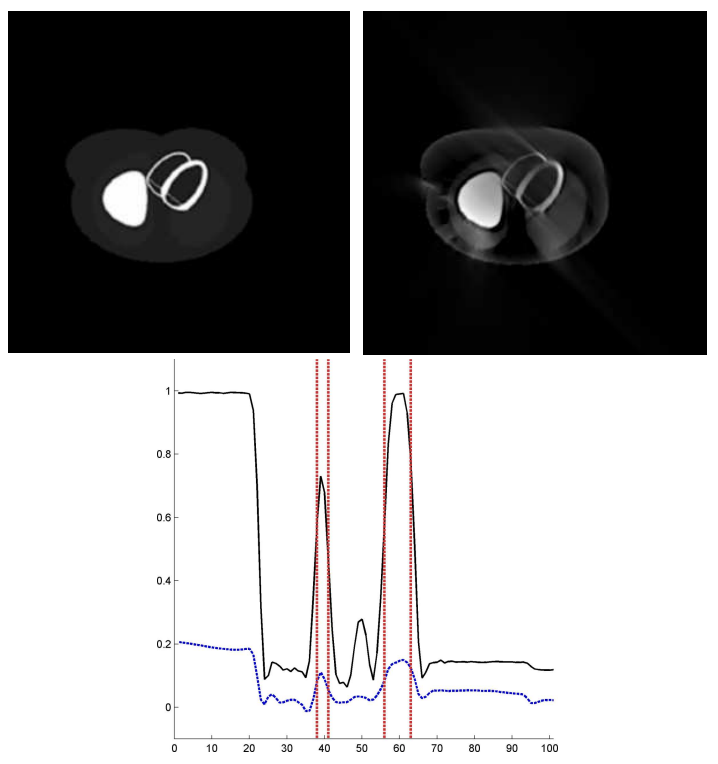

Figure 14: Comparison between non attenuated (top left) and attenuated (top right) reconstruction of the activity distribution. In the figure on the right, the intensity is scaled by a factor of 3.8. The figure at the bottom shows a profile of samples of counts for the non attenuation case (black) and for the attenuation case (blue).
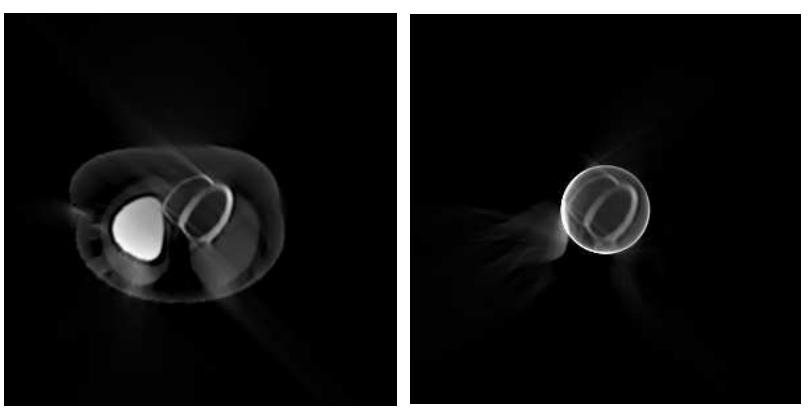

Figure 15: Visualization of the artifacts for the truncation case. The picture on the left shows the reconstruction of the non truncated projections. The picture on the right shows the reconstruction of the truncated projections. The intensity in the left picture is scaled by a factor of 3.7 and the intensity in the right picture is scaled by 2.50 .

large increase in counts at the boundaries but this artifact doesn't spread through the heart wall.

Finally, the characterization of the non-linear error is performed by measuring the ratio of count change between septal and lateral wall. Two sections are defined where the values are taken in Fig. 17 where the septum is the red region and the lateral wall is the green region. The septum is taken as reference and the average value of the septal wall is compared to the average value on the lateral wall. The calculation was performed and the septal-to-lateral wall count 

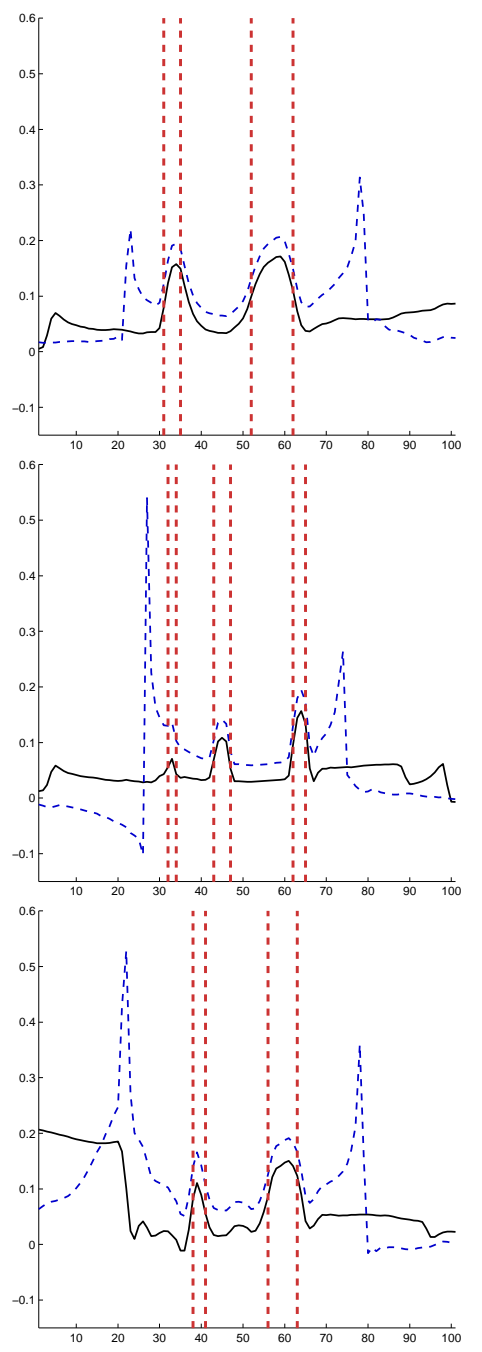

Figure 16: Sampling along the horizontal is green profile (at the top), along the diagonal is the yellow profile (picture in the middle) and along the vertical is the blue profile (bottom). The black line is the reconstructed counts for the attenuation but non truncated case and the blue dashed line shows the reconstruction of the attenuated truncated projections. The dashed red lines are the limits of the heart wall.

ratio was $5.5 \%$.

\subsubsection{Component}

The artifacts have the same behavior as in the non attenuation case. The majority of the error seems to be due to an incorrect DC component. A scaling factor would also be able to decrease the count mismatch on the heart wall.

In this case we applied a scaling factor $\mu=0.77$. The results of new samples are shown in Fig. 18. Therefore, using Eq. 4 and 5 and scaling the maximum intensity to one, the two error results were $d_{1} \simeq 0.17$ and $d_{2} \simeq 0.05$ for the entire heart and

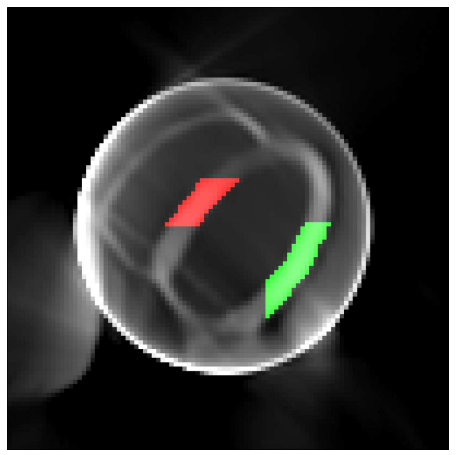

Figure 17: Mask used to calculate the septal-to-lateral wall count ratio where the septum is defined as the red region and the lateral wall as the green region.

$d_{1} \simeq 0.14, d_{2} \simeq 0.3$ for the left ventricle

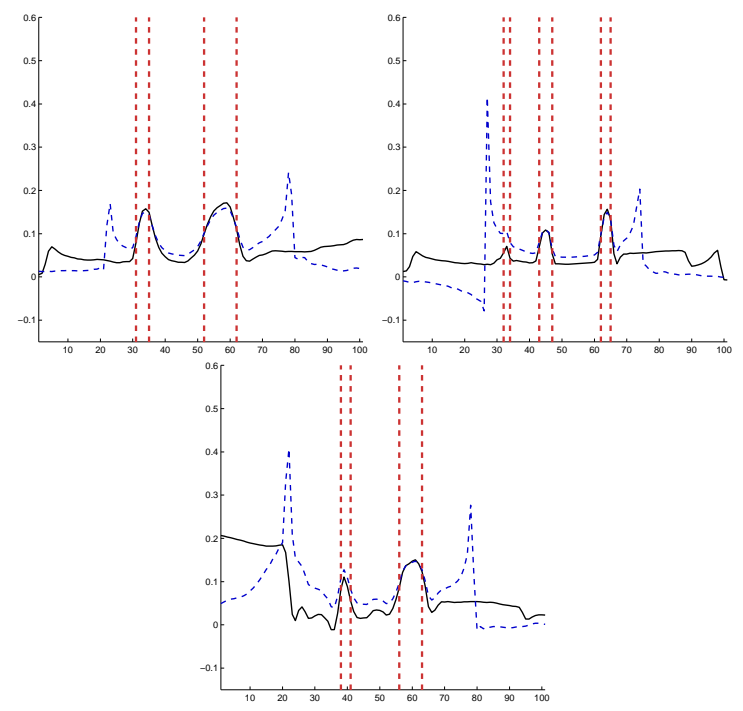

Figure 18: Samples for the corrected case with a scaling factor $\mu=0.77$. Horizontal is the green profile (first picture), diagonal is the yellow profile (picture in the middle) and vertical is blue profile (bottom picture). The black line is the reconstructed counts for the attenuation but non truncated case and the blue dashed line shows the reconstruction of the attenuated truncated projections. The dashed red lines are the limits of the heart wall.

The errors do not seem to get worse with attenuation. The majority of the mismatch corresponds to a problem of the scaling factor. The difference map of the heart wall is shown in Fig. 19. The error caused by the truncation is still an increase in the counts situated inside the ROI. The scaling factor decreases this difference especially on the left ventricle far from the liver. 

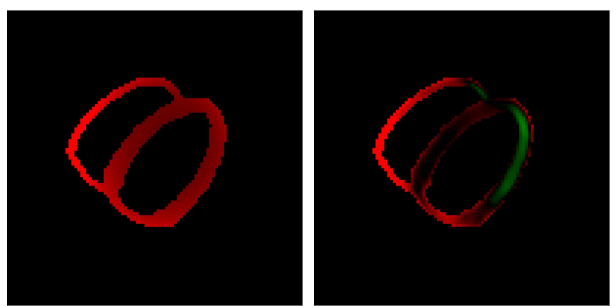

Figure 19: Error map for the attenuation case. The figure at the left shows the error map for the case without scaling and the pictures at the right shows the case with scaling by a factor $\mu=0.77$ applied to the reconstruction of truncated projections. The red color indicates an excess of counts due to the truncation, while the green shows a lack of it.

\subsection{Effect of the Additional Hot Struc- ture}

In this study, the attenuation is always taken is account in simulating the projections and the effect of the addition of a hot structure on the heart wall is studied. The goal is to determine if truncation increases the artifacts caused by the additional structure on the heart wall.

\subsubsection{Artifact Visualization}

The reconstruction was performed with and without the hot spot for the non truncated and truncated cases. The reconstructed activity maps are shown in Fig. 20.
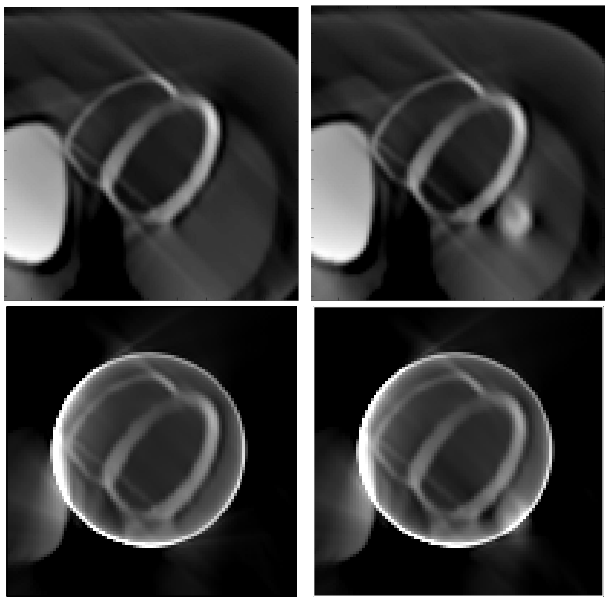

Figure 20: Effect of the hot spot. The pictures on the top show the results for the non truncated case. The left figure is the reconstruction without the hot spot and the right figure is the reconstruction with the hot spot. In the same order, the pictures on the bottom show the reconstruction for the truncation case.

\subsubsection{Artifact Quantification}

It is then interesting to study the quantification of samples on the region of the left ventricle close to the hot spot. Fig. 21 shows the results of sampling along the same lines for the non truncated case. The comparison is performed between the image without the hot structure (black line) and the image with it (blue line). The presence of the hot spot can be seen outside of the heart wall by the increased counts in the profile through the hot spot.

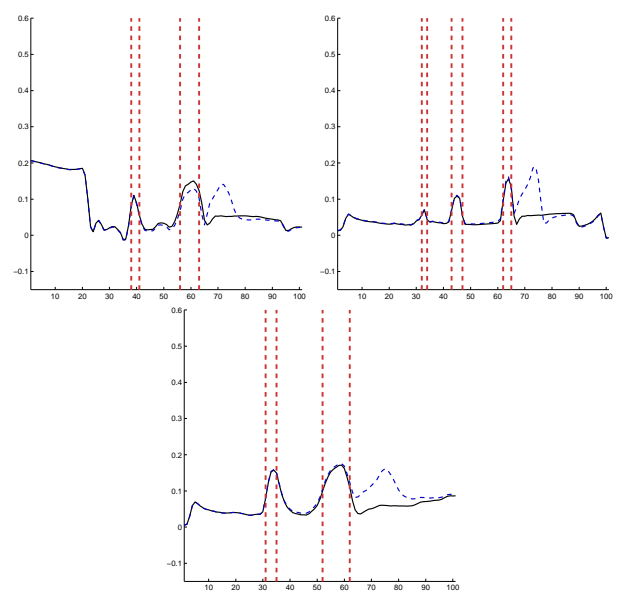

Figure 21: Profiles through the hot structure for the case without truncation. The profiles for the horizontal, oblique, and vertical lines are top left, top right and bottom figure, respectively. The plain black line is the reconstruction of the activity without the hot-spot and the dashed blue line is the one with the hot spot.

It is appreciated that the change induced on the wall (effect due to the attenuation) is very small even for regions close to the structure.

A second comparison was performed by reconstructing truncated projections and then checking to see if the artifacts became worse on the wall. These results are shown in Fig. 22. The black line still shows the reconstruction without the hot-structure and the blue one is the reconstruction with it. The ring artifact occurs exactly at the position of the hot spot. However, the significant decrease in the extra counts doesn't spread the artifacts to the heart wall, which would lead to a larger error.

\subsubsection{Error Comparison}

Both cases seem to produced small errors on the heart wall. The errors were calculated in the same way as before using Eq. 4 but this time the reconstructions were compared with and without the hot structure.

In the non truncated case, the error is $d \simeq 1.1 \%$ over the entire heart with an error of less than four 


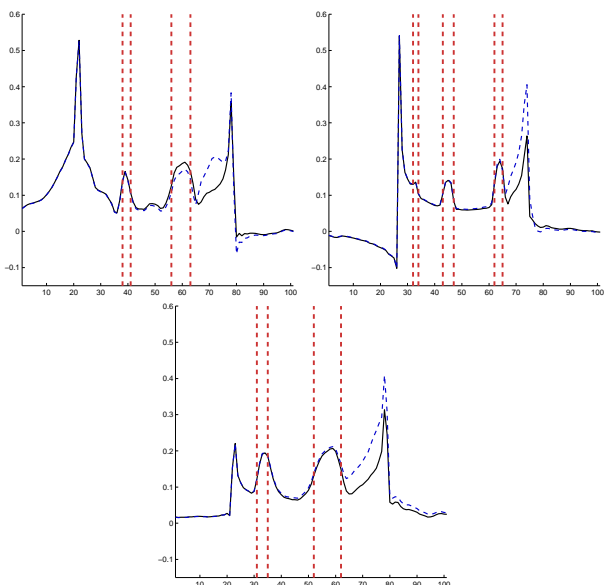

Figure 22: Profiles through the hot structure for the case of truncation. The profiles for the horizontal, oblique, and vertical lines are top left, top right and bottom figure, respectively. The plain black line is the reconstruction of the activity without the hot-spot and the dashed blue one with the hot spot.

percent close to the structure in the left ventricle. In the truncation case, the error is decreased to $d \simeq 0.35 \%$. Therefore the truncation doesn't make the distribution of the artifacts to become worse, but instead the effect is decreased.

Interestingly, the error map for the heart wall is exactly the same for the two cases as shown in Fig. 23 (the intensity scaling is different but the artifacts due to the hot-structure are the same). It can be noticed

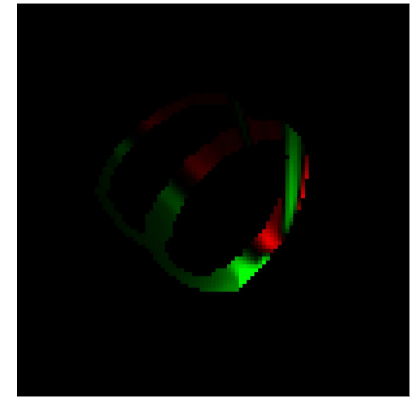

Figure 23: Error map for the effect of the hot structure near the heart wall. The map is exactly the same for the truncated and non truncated cases (if the intensity is scaled to its maximum). The red color indicates counts over for the activity and the green color indicates counts under due to the addition of the hot structure.

that an increase of counts should be expected due to the addition of a hot structure. However, the attenuation creates also a decrease of counts in the lateral part of the hot spot. In order to understand the difference due to artifacts generated by the hot spot, the error map is drawn on the entire surface (not lim- ited to the heart wall). The differences between no hot spot and hot spot are shown in Fig. 24. The dif-
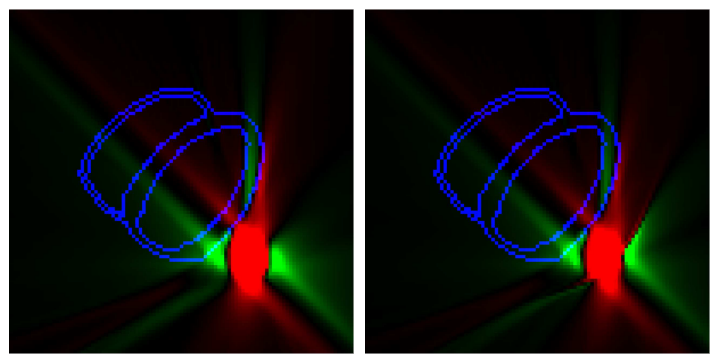

Figure 24: Error map for the effect of the hot structure on the entire surface. The figure on the left shows the difference due to the hot spot in the non truncation case. The figure on the right is for the truncation case. The red color is an increase of counts due to the hot structure while the green color is a decrease.

ference of artifacts due to truncation can be seen as acting only at the limits of the boundary of the ROI. The phenomenon does not spread through the wall of the left ventricle.

\subsection{Ring Artifact}

The largest artifact is indeed the ring effect with a large count over the true value at the truncation edge. Ways to reduce the artifact can be considered by looking at the sinogram. The ring effect is caused by applying a high frequency filter to the discontinuity at the truncation edge. Then an easy way to decrease this effect is to move the discontinuity away from the ROI.

One method would be to copy the value $p_{\theta}\left(r_{0}\right)$ and $p_{\theta}\left(-r_{0}\right)$ for larger $t$. Then the discontinuity will be translated outward and thus increase the radius of the ring. Therefore it will be further from the heart as seen in Fig. 25.

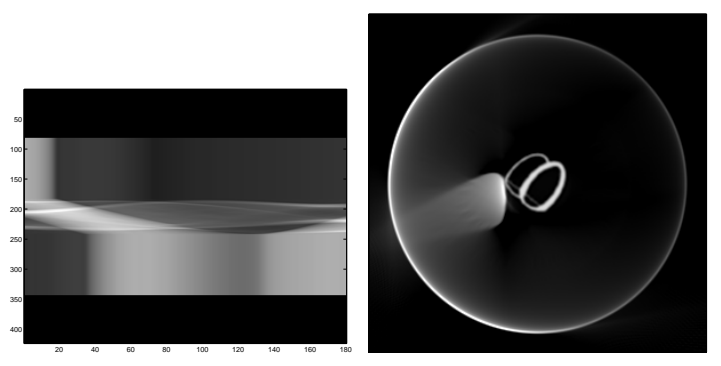

Figure 25: The picture on the left shows the copy of the line of the sinogram to translate the discontinuity. The picture on the right is the reconstuction where the ring effect is now far from the heart.

A better improvement is to suppress the ring di- 
rectly by extrapolating the value of the sinogram smoothly to zero [4]. Linear extrapolation is sufficient to suppress the ring artifact completely as seen in Fig. 26. In this case the interpolation function is $\frac{\left|s-s_{0}\right|}{s_{\max }}$ where $s_{\max }$ is the distance beyond which the values on the sinogram are all zero.
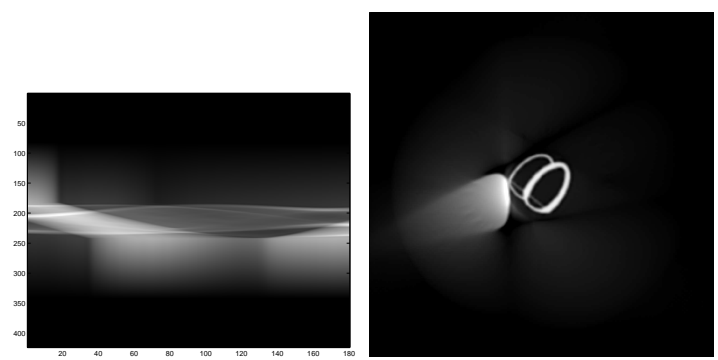

Figure 26: The ring effect is suppressed by linear interpolation of the values of the sinogram between the limit of the boundary to zero. The picture on the left is the sinogram and the picture on the right shows the reconstruction without the ring artifact.

Some improvement can still be made using a better approximation to match the derivative. For instance taking an interpolation with a cosine function $\cos ^{2}\left(\frac{\left|s-s_{0}\right|}{s_{\max }} \frac{\pi}{2}\right)$ instead of a linear function enables a smother transition at the end as seen in Fig. 27
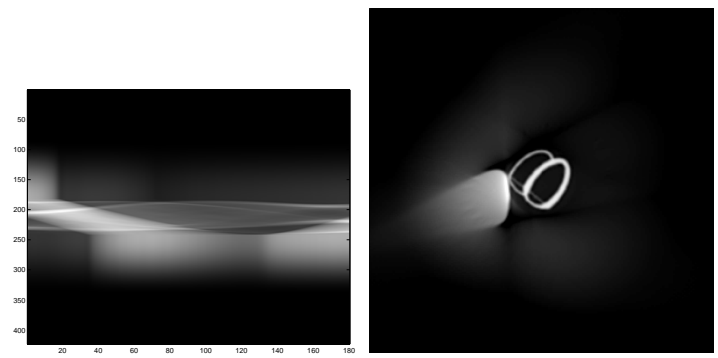

Figure 27: The ring effect is suppressed by interpolation with a cosine function for the values of the sinogram between the limit of the boundary to zero. The picture on the left is the sinogram and the picture on the right shows the reconstruction without the ring effect.

Finally the ring artifact can be described by the reconstruction given by the Hilbert Transform [3]. In the non attenuation case, the inverse Radon transform is given by

$$
r\left(p_{\theta}\right)=\mathcal{B}\left(\frac{\partial p_{\theta}}{\partial s} * h\right) .
$$

where $\mathcal{B}$ is the backprojection operator previously defined and $h: s \mapsto \frac{1}{2 \pi^{2} s}$. Then for the truncation case:

$$
r\left(p_{\theta}^{T}\right)=\mathcal{B}\left(\frac{\partial}{\partial s}\left(p_{\theta}(s) \Pi\left(\frac{s}{r_{0}}\right)\right) * h(s)\right) .
$$

Taking the derivative in the sense of a distribution gives:

$$
\begin{aligned}
\Rightarrow r\left(p_{\theta}^{T}\right)= & \mathcal{B}\left(\left[\Pi\left(\frac{s}{2 r_{0}}\right) \frac{\partial p_{\theta}}{\partial s}+\right.\right. \\
& p_{\theta}\left(-r_{0}\right) \delta\left(s+r_{0}\right) \\
& \left.\left.-p_{\theta}\left(r_{0}\right) \delta\left(s-r_{0}\right)\right] * h(s)\right) .
\end{aligned}
$$

Using the linearity of the backprojection operator $\mathcal{B}$ and the propriety of the dirac distribution gives the following:

$$
\begin{aligned}
\Rightarrow r\left(p_{\theta}^{T}\right)= & \mathcal{B}\left(\left(\Pi\left(\frac{s}{2 r_{0}}\right) \frac{\partial p_{\theta}}{\partial s}\right) * h(s)\right) \\
& +\mathcal{B}\left(p_{\theta}\left(-r_{0}\right) h\left(s+r_{0}\right)\right) \\
& +\mathcal{B}\left(p_{\theta}\left(r_{0}\right) h\left(s-r_{0}\right)\right) .
\end{aligned}
$$

The first term is the reconstruction without the ring effect and the last two terms incorporate the effect of the ring as they take into account the derivative of the $\Pi$ function. Therefore the ring effect (for the non attenuation case) takes into account only the projection value at $r_{0}$ and $-r_{0}$.

The two terms incorporating the ring effect are drawn in Fig. 28. The sum of the two terms gives the ring (drawn in Fig. 29) and the first term of Eq. 8 corresponds to the reconstruction without the ring.
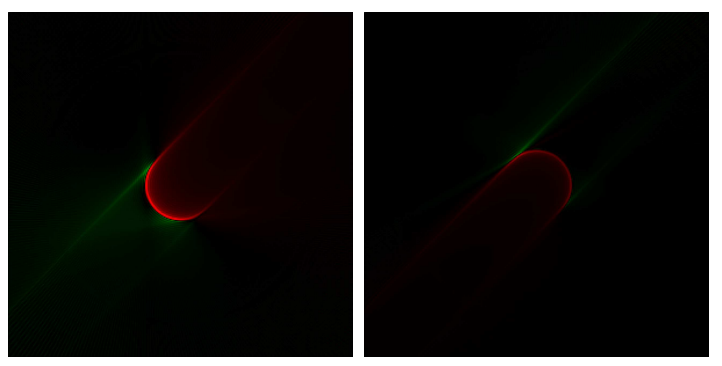

Figure 28: Visualization of the two terms of Eq. 8 showing the ring artifact. The picture on the left shows the first term and the figure on the right corresponds to the second term
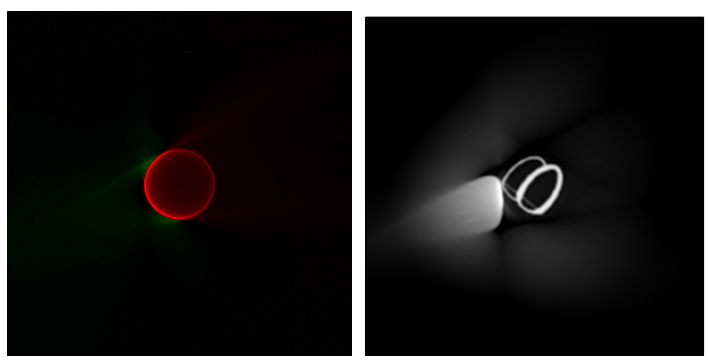

Figure 29: On the left visualization of the ring alone (sum of the two previous pictures) and on the right the reconstruction without the ring. 


\section{Algebraic Methods}

So far, only reconstruction with filtered backprojection has been considered. Here a comparison between FPB and an algebraic reconstruction method will be performed. For an algebraic reconstruction, the projection matrix $W$ needs to be constructed. Denoting $p$ as the vector of projections and $x$ the unknown vector of the three-dimensional activity distribution, the goal is to solve for $x$ in the linear system of equations $W x=p$. In the case of truncated projection, the vector $p$ is truncated by keeping only the projection values inside the ROI. But the matrix $W$ is also modified to take in account this lack of projection measurements and the rows for the positions outside of the ROI are deleted. Two algebraic reconstruction methods were considered. The least square method was used to invert $W$ to give a minimum residual of $W x-p$ in a least square sense. The Algebraic Reconstruction Technique (ART) was also used to reconstruct the original distribution by succesive projections. The study was performed without including attenuation in the projections and with the same conditions used in the experiment for the study of FBP. The reconstruction is shown in Fig. 30. The
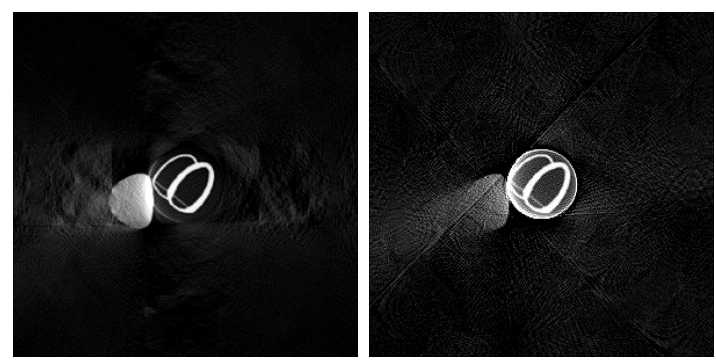

Figure 30: Reconstruction with algebraic methods. The figure on the left is the reconstruction with the least square method. The figure on the right is the reconstruction with the ART algorithm.

ART reconstruction seems to have the same characteristics as the FBP reconstruction. The ring effect is still present and the region outside of the ROI has a smaller value than the region inside. The reconstruction is also nosier and a smoothing filter should be used for eliminate the high frequency components. The least square method gives different characteristics. The reconstruction doesn't produce the ring artifact and the regions outside of the ROI are also reconstructed. Less artifacts seem to be present than in the FBP reconstruction.

A quantitative study was also performed to see if the truncated reconstruction deforms the image or changes its average value. The sampling for the two methods was performed along the green vertical line and the result is shown in Fig. 31.

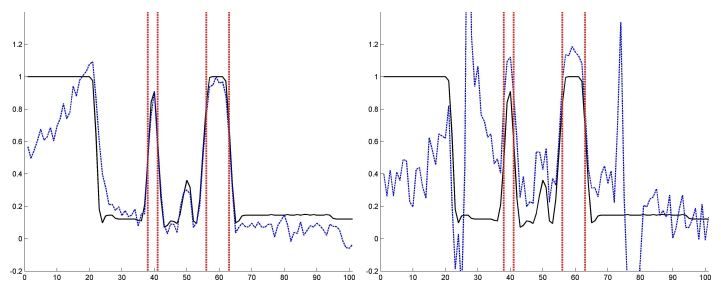

Figure 31: Sampled profiles of the algebraic reconstruction. The black line is the true value and the dashed blue line is the reconstructed value. The vertical red lines are the boundaries of the heart wall. The picture on the left is the results for the least square reconstruction. The results on the right are the reconstruction for the ART reconstruction.

The ART reconstruction is noisy and presents characteristics of high values along the ring of the truncation and a global increase of the average value inside the region of interest. The reconstruction with the least square method however is very close to the true value. The curve is almost not deformed and the absolute count value is unchanged, implying that the DC component is correctly reconstructed. Outside of the ROI, the values are noisier due to the fewer number of measurements, but the truncated values follow correctly the closest part of the liver. For larger radius, the counts decrease and the value is then not correctly reconstructed, but the error is less than with the FBP method. The least square method seems to be far more accurate than the FBP reconstruction. Moreover, the accuracy of the reconstruction depends theoretically on the number of projections that can give information for the reconstruction of each voxel. Therefore, the precision of the reconstruction can come arbitrarily close to the real value independent of the radius of the truncation. If the data are consistent and sufficient to make the matrix surjective, the reconstruction can then be exact. In Fig. 32, the reconstruction is performed with 1000 projections sampled. The reconstruction is almost perfect even in the truncated region.

However the method of least squares is very sensible to inconsistent data and therefore depends on the noise level. This problem exists already for non truncated data where the least squares method is also sensible to noise. For the case of truncation, the method is even more sensitive to noise due to the few measurements outside of the ROI, and cannot reconstruct correctly the value in presence of noise. An example is given with the same conditions but taking 400 projections and adding Gaussian noise to the projection data with $\sigma=5 \% p_{\theta}$. The noisy sinogram and the least square reconstruction are shown in Fig. 33. The 


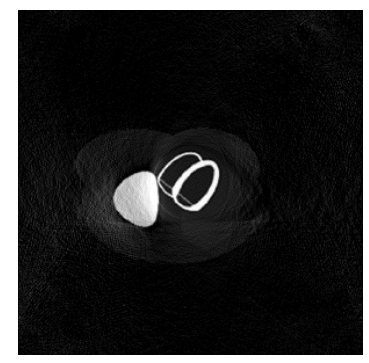

Figure 32: Reconstruction of 1000 projection angles using the least square method.

reconstruction is completely wrong since the data are not anymore consistent.
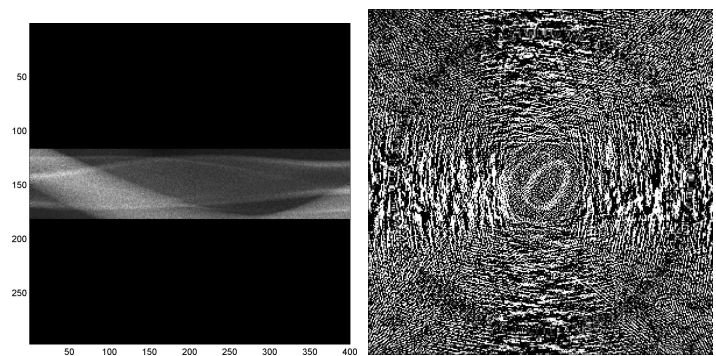

Figure 33: The picture on the left is the noisy sinogram with $\sigma=5 \% p_{\theta}$. The picture on the right is the reconstruction of the noisy data with the least square method.

\section{Discussion}

For a rotation of the camera spanning $180^{\circ}$ a study was performed to quantify the effect of truncation on a region close to the wall of the heart. It was shown that the truncation has very little effect on the wall of the left ventricle.

The effect of attenuation has, by far, a much more significant effect on the clinical interpretation of the reconstruction. The major effect is due to a scaling of the DC component which doesn't produce a relative intensity error in the same slice, but it can produce a problem in a slice to slice count variation as the truncated geometry varies. The error in counts can be reduced by a homogeneous scaling factor, which is unknown and depends on the activity map as well as on the attenuation distribution. However, the scaling factor can roughly be approximated by knowing a apriori an estimate of these two distributions.

It is noticed that the truncated reconstruction gives an increase of counts inside the ROI. This is due to the filtered backprojection algorithm. When there is activity in the projected data outside the area of the
ROI, the filtered backprojection algorithm will redistribute these counts to inside the smaller truncated area. Thus, the activity is higher than the truth and it depends on the area and distribution of the truncated activity.

The truncation artifacts depend on the reconstruction algorithm used. In most of our work, the Filtered Backprojection (FBP) algorithm was used. Its characteristics are the presence of the ring artifact at the boundary of the truncation and approximately null values outside of the ROI. It was shown that this ring artifact can be eliminated easily with only the knowledge of the projection data at the boundary. Of course the attenuation effect makes this correction more complex from a mathematical point of view. However, it was shown that there is little difference in the results between using a simple interpolation beyond the truncation edge and an exact elimination of the ring.

It was shown in some examples that even if attenuation is mixed with truncation, the artifacts due to the truncation do not spread more inside the ROI. This was especially true for the additional small hot structure. This structure has a very small area and does not contribute many counts from the attenuation and truncation point of view. The effect is different for the liver which has a large area, where there is more lost of information and artifacts are spread onto the right ventricle. In a study focused on the right ventricle, this truncation could cause more problems for clinical diagnosis.

Lastly, a comparison with algebraic reconstruction methods was performed. The least squares solution seems to be far more accurate than the FBP reconstruction and can even reconstruct the region outside of the ROI. However the method is also sensitive to noise and failed completely as soon as the data were not consistent. This is due to the fact the method does not take into account any minimization of energy for the smoothness of the picture. Methods such as OSEM which model the counts noise inside the reconstruction process are able to deal with the noise level and also perform some truncated reconstruction [1] with better quality than FBP.

\section{Acknowledgement}

This work was supported in part by the Director, Office of Science, Office of Biological and Environmental Research, Medical Science Division of the U.S. Department of Energy (DEO) under Contract No. DEAC02-05CH11231 and in part by Public Health Service (NIH) grant number R01 EB000121 awarded by the National Institute of Biomedical Imaging and Bio- 
engineering, Department of Health and Human Services.

\section{References}

[1] Georges K. Gregoriou, Benjamin M.W. Tsui, and Grant T. Gullberg. Effect of Truncated Projection on Defect Detection in Attenuation Compensated Fanbeam Detection in Attenuation Compensated Fanbeam Cardiac SPECT. Nuclear Medicine, 39(1):166-175, January 1998.

[2] Frédéric Noo and Jean-Marc Wagner. Image Reconstruction in 2D SPECT with $180^{\circ}$ Acquisition. Inverse Problems, 17:1357-1371, August 2001.

[3] A.G. Ramm and A.I. Katsevich. The Radon Transform and Local Tomography. CRC, 1995.

[4] Maria Magnusion Seger. Rampfilter Implementation on Truncated Projection Data. Application to 3D Linear Tomography for Logs. In Proceedings SSABO2 Symposium on Image Analysis, pages 33-36. SSAB, March 2002. 